\title{
INTRAVENTRICULAR TEICOPLANIN
}

\author{
Shanti $R^{1}$, Sam IC ${ }^{2}$, Hany Ariffin ${ }^{1}$ \\ ${ }^{1}$ Department of Paediatrics, Faculty of Medicine, University of Malaya, Kuala Lumpur \\ 2 Department of Medical Microbiology, Faculty of Medicine, University of Malaya, Kuala Lumpur
}

\begin{abstract}
:
Following craniotomy for an atypical rhabdoid tumour of the posterior cranial fossa, a fourteenmonth-old boy developed a ventriculitis with methicillin resistant Staphylococcus epidermidis (MRSE) which is associated with the use of a ventriculoperitoneal shunt. Treatment with intravenous vancomycin resulted in a severe allergic skin reaction. Substitution with intravenous teicoplanin resulted in negative blood culture and MRSE ventriculitis was successfully eradicated with concomitant use of intraventricular teicoplanin. No signs of recurrent infection or adverse events occurred. Intraventricular teicoplanin is safe and effective for the treatment of staphylococcal neurosurgical shunt infections. (JUMMEC 2009; 12(1):35-38)
\end{abstract}

KEYWORDS: teicoplanin, ventriculitis, neurosurgical shunts

\section{Introduction}

Neurosurgical shunt infections can be insidious with the incidence ranging from $1 \%$ to $39 \%$ (1). Coagulase-negative Staphylococcus (CoNS) is the most frequently isolated organism (2). CoNS is a normal inhabitants of the human skin and mucous membranes. Patients most at risk for CoNS infection frequently have a disruption in their host defense mechanisms due to surgery, foreign body placement, immunosuppression or a combination of these factors. CoNS is able to adhere to and grow on the surface of plastic catheters and produce protective extracellular material (slime) that covers the bacterial cells.

A common approach to the treatment of neurosurgical shunt infections includes the removal of the infected shunt with implantation of an external ventricular drainage device, usually in combination with appropriate antimicrobial chemotherapy (1). The choice of antibiotics in this setting depends not only on the susceptibility of the pathogen, but also on the ability of the drug to cross the blood brain barrier.

Vancomycin is highly efficacious against CoNS and is a valuable drug for treatment of implant deviceassociated infections (3). If the causative organism is a CoNS, the patient should be treated by instilling vancomycin (5-20 mg, depending on ventricular volume). For example, $5 \mathrm{mg}$ for patients with 'slit' ventricles, $10 \mathrm{mg}$ for those with ventricles of normal size and $15-20 \mathrm{mg}$ for those with greater than normal volumes directly into the ventricles and clamping the drain for approximately $15 \mathrm{~min}$ (4). If the cerebrospinal fluid (CSF) is draining freely ( $>100 \mathrm{ml} /$ day), a daily dose should be administered, but if there is no or little CSF drainage $(<50 \mathrm{ml} /$ day $)$, the dose need to be repeated only once every third day; patients from whom intermediate volumes of CSF $(50-100 \mathrm{ml} /$ day $)$ are draining should be given doses on alternate days and those from whom very large volumes (>200 ml/day) are draining may require higher dosages or twice daily instillation (4). However, vancomycin has a number of potentially serious side effects like "Red-man Syndrome," a severe allergic reaction. Teicoplanin, another glycopeptide, offers comparable efficacy with fewer side effects (5). In addition, the pharmacokinetics of teicoplanin allows its administration in a single daily dose.

Here, we report our experience with the intraventricular administration of teicoplanin for a recalcitrant neurosurgical shunt infection after initial vancomycin therapy was precluded by serious side effects.

Correspondence:

Shanti Ramachandran

Department of Paediatrics

Faculty of Medicine, University of Malaya

50603 Kuala Lumpur, Malaysia

Email: rshanti@um.edu.my 


\section{Methods}

A previously well 14-month-old boy (body weight of $10 \mathrm{~kg}$ ) was presented to the University of Malaya Medical Centre, Kuala Lumpur. He had recurrent vomiting for two weeks and an afebrile seizure for one day. MRI brain and spine showed a large posterior fossa tumour compressing the cerebellar vermis posteriorly, and the brain stem and the fourth ventricle anteriorly. An extraventricular drain (EVD) was inserted then.

Near total removal of the tumour was done three days later and the histopathological examination showed an atypical rhabdoid tumour of the posterior fossa (WHO Grade IV). The EVD was removed three weeks later. The CSF examination was clear then. A central venous line was inserted for commencement of chemotherapy.

A week following central venous line insertion, he developed a high grade fever and had abnormal movements of the limbs. CSF obtained from an intraventriculartap showed glucose of $2.0 \mathrm{mmol} / \mathrm{I}$ (serum glucose was $5.4 \mathrm{mmol} / \mathrm{l})$, total protein of $0.5 \mathrm{gm} / \mathrm{L}$ and leucocyte count of $40000 / \mathrm{mm} 3$ (100\% polymorphs). The CSF culture was sterile then, but methicillin resistant Staphylococcus epidermidis (MRSE) was cultured from the blood. He was started on intravenous vancomycin. However, this led to him developing a generalized exanthema with hypotension, known as the Red Man Syndrome, a condition associated with histamine release after intravenously administered vancomycin (6). This complication persisted despite administering vancomycin at a slower rate and with antihistamine prophylaxis. Therefore, intravenous vancomycin was substituted with intravenous teicoplanin.

His blood culture became sterile five days after intravenous teicoplanin, the CSF C\&S remained sterile but the CSF FEME showed persistence of low glucose and leucocytosis.

On day ten of the illness, he developed another episode of seizure. This time Cushing's reflex was elicited. An urgent CT scan of the brain showed an enlarging tumour with acute hydrocephalus. An EVD was inserted and this was converted to a ventriculoperitoneal shunt two days later. The first course of chemotherapy was commenced and intravenous teicoplanin was continued as he remained febrile. Unfortunately, the ventriculoperitoneal shunt was complicated with ventriculitis. The CSF FEME showed glucose of $2.1 \mathrm{mmol} / \mathrm{l}$, protein of $1.43 \mathrm{~g} / \mathrm{L}$, leucocytes count of $643000 / \mathrm{mm} 3$ (100\% polymorphs). This led to it's removal. Ten days post-chemotherapy he became neutropenic and this time, CSF culture yielded MRSE.

He had weekly CSF cultures done which showed MRSE ventriculitis persisted in five consecutive CSF C\&S despite five weeks of intravenous teicoplanin. Hence intraventricular teicoplanin $10 \mathrm{mg}$ daily was added. The EVD was clamped for two hours after instillation of intraventricular teicoplanin. Every four days, CSF was obtained from the EVD and was analysed for chemistry and culture. The CSF FEME showed improving infection parameters and CSF culture became sterile after four days of intraventricular teicoplanin (Figure 1).

His fever settled after one week of intraventricular teicoplanin and subsequent CSF and blood cultures remained sterile. He received two weeks of intraventricular teicoplanin and seven weeks of intravenous teicoplanin in total.

He had a repeat MRI Brain one week after completion of antibiotic therapy which showed an enlarging tumour. Hence, a second course of chemotherapy was administered. The EVD remained in situ. He had severe febrile neutropenia one week upon completion of second chemotherapy and finally died of septicaemia. His repeated blood and CSF C\&S during this period of time remained sterile and there were no recrudescense of MRSE seen in these cultures.

\section{Discussion}

Teicoplanin was first discovered in 1978 by fermentation of Actinoplanes teichomyceticus. Like vancomycin, teicoplanin is a glycopeptide and is active against gram-positive organism infections, including MRSE. It interferes with cell wall synthesis by inhibiting polymerization of peptidoglycan and has bioavailability of $90-95 \%$ with a half life as long as 100 hours in patients with normal renal function. However, when given intravenously, teicoplanin penetrates slowly and poorly into the CSF, although penetration is enhanced by meningeal inflammation. This explains why we were not able to eradicate MRSE ventriculitis in this patient, despite five weeks of intravenous teicoplanin therapy. The intraventricular 


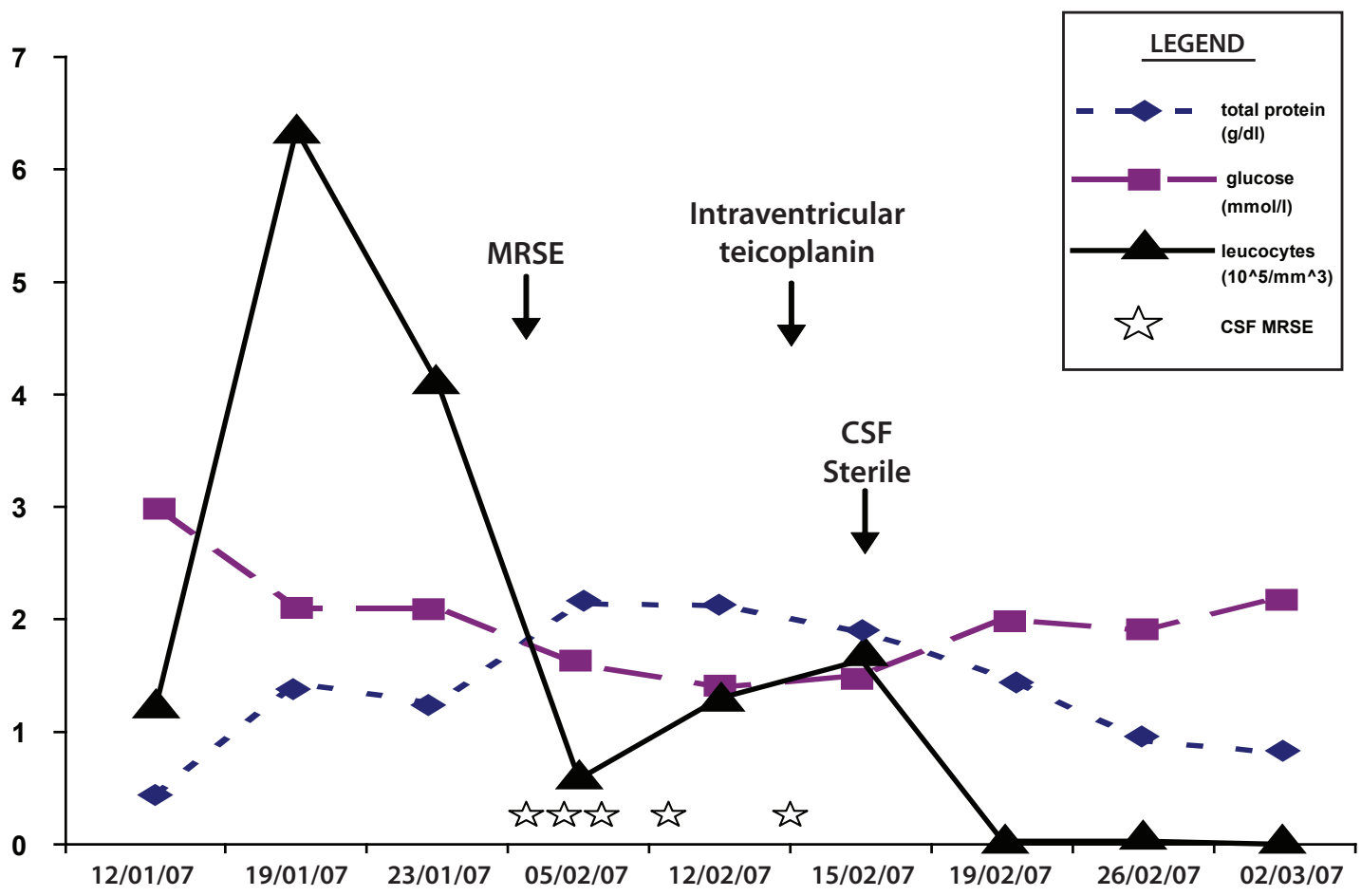

Figure 1: Graph showing CSF analyses and cultures response to intraventricular teicoplanin

administration of teicoplanin resulted in immediate sterilization of CSF. It would have been helpful if we have the facilities of determining the peak and trough levels of teicoplanin in both the CSF and blood to affirm its efficacy in this patient. However, it is known that after intraventricular administration, high and prolonged levels of teicoplanin are found in the CSF (1).

James et al demonstrated in 37 patients, aged between one month and 12 years, that in patients who were receiving maximal intravenous antibiotic therapy alone, CSF concentration were below $5 \mu \mathrm{g} / \mathrm{ml}$, whereas those receiving antibiotics through direct ventricular puncture-EVD, or a shunt reservoir usually had concentrations over $5 \mu \mathrm{g} / \mathrm{ml}$. To obtain a high concentration of an antibiotic in the CSF, one should administer it directly into the ventricle (7).

In other literature, 17 patients with direct CSF administration of teicoplanin are described (8). Of these, three were infants, three children and eleven adults. In nine cases, the isolated organism was CoNS. In the infants, $5 \mathrm{mg}$ intraventricular teicoplanin was used, in children $10 \mathrm{mg}$ was used and most adults were treated with $20 \mathrm{mg} /$ day. Concomitant intravenous teicoplanin was continued. Duration of treatment ranged from 6-25 days (mean 16 days) with eradication achieved between two and severn days (median 4.4 days). In general, teicoplanin was well tolerated and all 17 patients were cured. The alternate daily dose was as effective as the daily regime.

In this patient of 14 months of age with rhabdoid tumour of the posterior fossa, intraventricular teicoplanin with concomitant intravenous teicoplanin had successfully eradicated MRSE ventriculitis without any serious adverse reactions. Teicoplanin may be an alternative to vancomycin for the antimicrobial therapy of CSF shunts infections especially in those with hypersensitivity to vancomycin as demonstrated in this patient.

\section{References}

1. Cruciani M, Navarra A, Di Perri G, Andreoni M, Danzi MC, Concia E, Bassetti D. Evaluation of intraventricular of teicoplanin for the treatment of neurosurgical shunt infections. Clin Infect Dis 1992;15(2): 285-289.

2. Garvey G. Current concepts of bacterial infections of the central nervous system. Bacterial meningitis and bacterial brain abscess. J Neurosurg 1983; 59: 735-744.

3. McLamin RL, Frame PT. Treatment of infections of CSF shunt. Rev Infect Dis 1987; 9: 595-603. 
4. Brown EM. Review article: The management of neurosurgical patients with postoperative bacterial or aseptic meningitis or external ventricular drainassociated ventriculitis. Br J Neurosurg 2000; 14(1): 7-12.

5. Wood MJ. The comparative efficacy and safety of teicoplanin and vancomycin. J Antimicrob Chemother 1996;37: 209-222.

6. Davis RL, Smith AL, Koup JR. The "Redman's Syndrome" and slow infusion of vancomycin. Ann Intern Med 1986;104: 255-286.
7. James HE, Wilson HD, Connor JD, Walsh JW. Intraventricular CSF antibiotic concentration in patients with intraventricular infections. Neurosurgery 1982; 10(1): 50-54.

8. Beenen LFM, Touw DJ, Hekker TAM, Haring DAJP. Pharmacokinetics of intraventriculary administered teicoplanin in Staphylococci ventriculitis. Pharm World Sci 2000; 22(4): 127-129. 